This is the final peer-reviewed accepted manuscript of:

Y. Iwamoto, R. Meattini, D. Chiaravalli, G. Palli, K. Shibuya and C. Melchiorri, "A Low Cost Tactile Sensor for Large Surfaces Based on Deformable Skin with Embedded IMU," 2020 IEEE Conference on Industrial Cyberphysical Systems (ICPS), Tampere, Finland, 2020, pp. 501-506, doi: 10.1109/ICPS48405.2020.9274737.

The final published version is available online at DOI: $10.1109 /$ ICPS48405.2020.9274737

Rights / License:

The terms and conditions for the reuse of this version of the manuscript are specified in the publishing policy. For all terms of use and more information see the publisher's website.

This item was downloaded from IRIS Università di Bologna (https://cris.unibo.it/)

When citing, please refer to the published version. 


\title{
A Low Cost Tactile Sensor for Large Surfaces Based on Deformable Skin with Embedded IMU
}

\author{
Yuki Iwamoto**, Roberto Meattini*, Davide Chiaravalli*, Gianluca Palli*, Koji Shibuya** and Claudio Melchiorri* \\ **Dept. of Mechanical and Systems Engineering \\ Ryukoku University, Otsu, Japan \\ koji@rins.ryukoku.ac.jp \\ ${ }^{*}$ Dept. of Electrical, Electronic and Information Engineering (DEI) \\ University of Bologna, Bologna, Italy \\ \{roberto.meattini2,davide.chiaravalli,gianluca.palli,claudio.melchiorri\}@unibo.it
}

\begin{abstract}
This paper deals with the design, fabrication and preliminary experimental results of a novel soft tactile sensing system for large surfaces, aiming at detecting the location of the contact points on the surface of the sensor. The sensor is composed by soft material with an IMU embedded on a deformable silicon-based surface. Using the data provided from the IMU during morphological variations of the soft sensor, the aim of the sensing system is to recognize different locations of single contact points and linear regions of contact points resembling the contacts with soft linear objects. In order to achieve this behaviour, an artificial neural network has been used, and evaluated trough experiments. The reported results show that the sensing system is able to discriminate between a grid of single contact locations, and among different linear regions of contact points, with a mean accuracy superior than $80 \%$, and peak accuracy of $97.97 \%$ (for the single single point contact locations) and $\mathbf{9 7 . 5 4 \%}$ (for the linear regions of contact points.)
\end{abstract}

Index Terms-Soft Robotics, IMU, Silicone, Tactile Sensor, Soft Materials.

\section{INTRODUCTION}

The field of Soft Robotics is rapidly expanding in recent years [1], thanks to many researchers working on a wide variety of soft robots. Soft Robotics can be roughly divided into two groups of applications, related to the study of actuators and sensors. In the field of the actuator, researchers have worked extensively in developing solutions for robotic manipulators and grippers, often basing their study on behaviours that can be found in nature, as for the case octopuses, snakes or caterpillars, and many others [2], [3]. For example, in [4], a soft gripper was actuated pneumatically, highlighting the simplification and effectiveness with respect to approaches based on electric motors.

With regard to sensing systems based on soft materials, most of the researchers have studied and developed devices that are strongly related to the field of the morphological

This research was partially supported by the Joint Research Center for Science and Technology of Ryukoku University and JSPS Grant-in-aid for Scientific Research (KAKENHI) grant number 1, and by the European Commission's Horizon 2020 Framework Programme with the project REMODEL - Robotic technologies for the manipulation of complex deformable linear objects - under grant agreement No 870133. computation [5]. In the present work, we also focus on this kind of sensing devices, in order to develop a soft tactile sensor, since the possibility of having an easy-to-build and low-cost sensing system of this type can be important in many robotics applications. Many researchers have developed a soft material based tactile sensor. Kadowaki et al. [6] developed a soft tactile sensor based on Light Emitting Diodes (LEDs) and light receiver elements. Paulino et al. [7] developed a lowcost soft tactile sensor for a robot finger,exploiting Hall-effect sensor. Hammond et al. [8] used liquid metal circuits in order to develop a soft sensorized glove for the measurements of the human hand motions and forces.

In this article, we propose a tactile sensor based on a silicone skin attached on a proper base in order to obtain a chamber. In this way, by inserting inserting air into the chamber, the silicone skin is inflated, changing its morphological structure and making the sensor able to work with both very soft and very hard objects. The present work is an advancement with the respect to our previous works. In [9], [10], we embedded a strain gauge in the silicon skin also reporting for its computational model. Subsequently, we developed a similar sensing system by embedding on the silicon skin an Inertial Measurement Unit (IMU) realized with a rigid PCB. This kind of sensor had the dimension of $64 \times 64 \times 25 \mathrm{~mm}$, and the presence of the rigid IMU-PCB limited the morphological deformations of the silicon, also causing damages of the soft skin after short usage. Furthermore, we just evaluated a very rough possibility of detecting tactile motions with such sensor [11], without studying the problem of contact points and regions detection.

In this paper, we improve the design, size and functioning of the sensing system with respect to our previous works. First of all, in this work a flexible PCB in the silicon-based skin of sensor is introduced, then the dimension of the sensor is also reduced. Finally, we evaluated via experimental sessions its accuracy in detecting points and linear contact regions. To achieve this goal, a specific setup has been developed: the soft sensor is equipped by a 6-axis Force/Torque (F/T) sensor, and a robot manipulator is used in order to have precise position and force measurements as ground truth. An artificial 


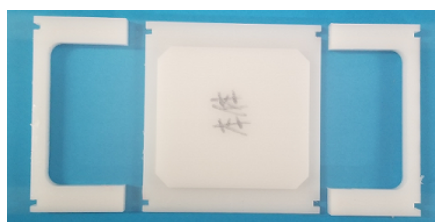

(a) Parts of mold

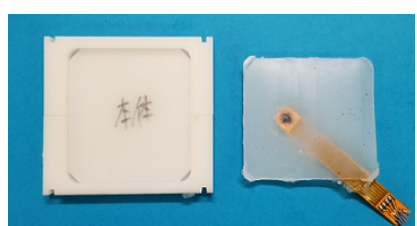

(b) Assembled mold and silicon skin.
Fig. 1. Mold for the silicon skin and cured silicon skin.

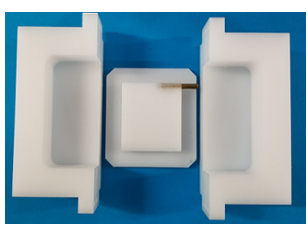

(a) Parts of mold.

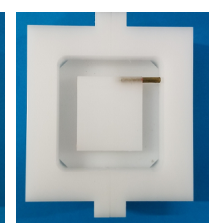

(b) Assembled mold.

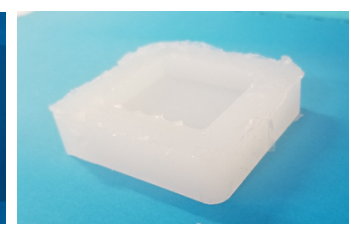

(c) Cured silicone base.
Fig. 2. Mold for the silicon base and cured silicon base.

neural network (ANN) is used to train a model used to obtain the output of the tactile sensor from the embedded IMU. In this way, we evaluated that the proposed sensor is capable of detecting contact point locations and discriminating between different linear contact regions resembling the contact with a thin objects with a mean accuracy superior than $80 \%$. The developed sensor can be then used to evaluate the orientation of a thin object, such as cable, over a large surface.

The article is organized as follows: Sec. II illustrated the materials and the fabrication techniques of the soft tactile sensor; in Sec. III the experimental setup is described together with the protocol for the evaluation of the system, also reporting for the obtained results and related considerations; finally, Sec. IV outlines the conclusions.

\section{Materials And Methods}

\section{A. Materials and Fabrication}

The material used to build the tactile sensor is silicon rubber (in detail, for the base: Dragon Skin30 (D30), for the skin: Ecoflex30 (E30) and Dragon Skin 10 (D10), available

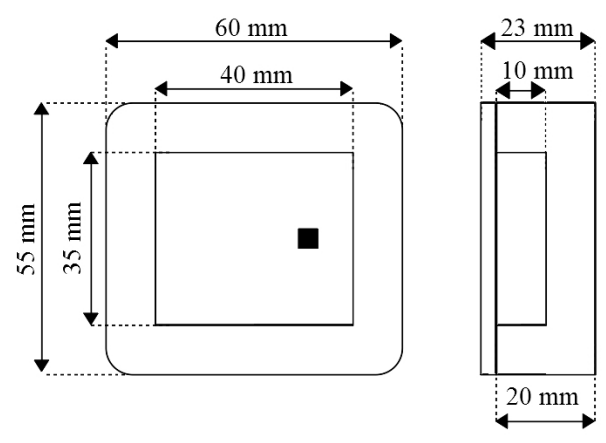

Fig. 3. Soft material tactile sensor.

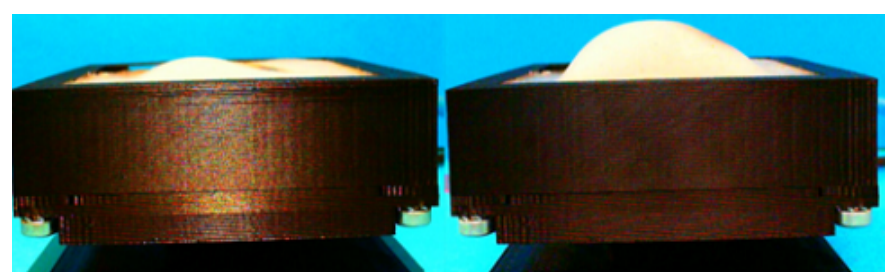

Fig. 4. Soft material tactile sensor.

from the company Smooth-On Inc ${ }^{1}$.). This material is selected because of its properties of being modelled very easily, which is suitable for the implementation of the deformable skin for our sensor.

For the skin implementation (see Fig. 1), two kind of liquid silicon - Ecoflex30 (E30) and Dragon Skin 10 (D10) - which have the same weight ratio, are mixed up. A mold is created by means of $3 \mathrm{D}$ printing (printer: Zortrax m200 plus, by Zortrax Inc ${ }^{2}$.). As mentioned in the introduction, in our previous work we embedded a rigid PCB accelerometer ( $4 \mathrm{~mm}$ of thickness) within the skin of the sensor, however in this way the accelerometer inhibited the full deformation of the silicone skin. Therefore, we chose in this work a flexible PCB (available from Shinwa Print Industry Co. ${ }^{3}$ ). In particular, this flexible PCB sensor has only $0.3 \mathrm{~mm}$ of thickness, embedding a flexible circuit and an IMU (the latter, with $2 \mathrm{~mm}$ of thickness.)

For the base implementation, we poured liquid silicone rubber to the mold illustrated in Fig. 2, which was made of polyoxymethylene (POM) thermoplastic. Thereafter, in order to remove several imperfections and bubbles the liquid silicon creates, we put the mold in a vacuum defoamer. After that, the liquid silicone was cured in an oven at the temperature of 70 degree Celsius. The aspect of the completed sensor's base can observed in Fig. 2(c).

The completed structure of the soft tactile system is shown in Fig. 3. The base has dimension of $60 \times 55 \times 20 \mathrm{~mm}$, whereas the dimension of the chamber is $35 \times 40 \times 10 \mathrm{~mm}$. The proposed soft tactile sensor is $30 \%$ smaller than the prototype presented in our previous works.

\section{B. Working Principle of the Sensor}

The working principle of the sensing system is based on the measurement of the contact surface deformation due to the contact with he external object through a 3-axis accelerometer. Moreover, the possibility of changing the pressure inside air chamber, as shown in Fig. 4, enables to change the sensitivity of the system, making it suitable for the interaction with a very large set of object, ranging from deformable, very soft and very fragile objects to very stiff ones. In the left side of Fig. 4, it is possible to observe the sensor configuration when the chamber is empty, i.e. the sensor is not inflated by air. Conversely, on the right side of Fig. 4, the sensor is inflated

\footnotetext{
${ }^{1}$ www.smooth-on.com

${ }^{2}$ www.3dpworld.it

${ }^{3}$ www.shinwa-print-ind.co.jp
} 


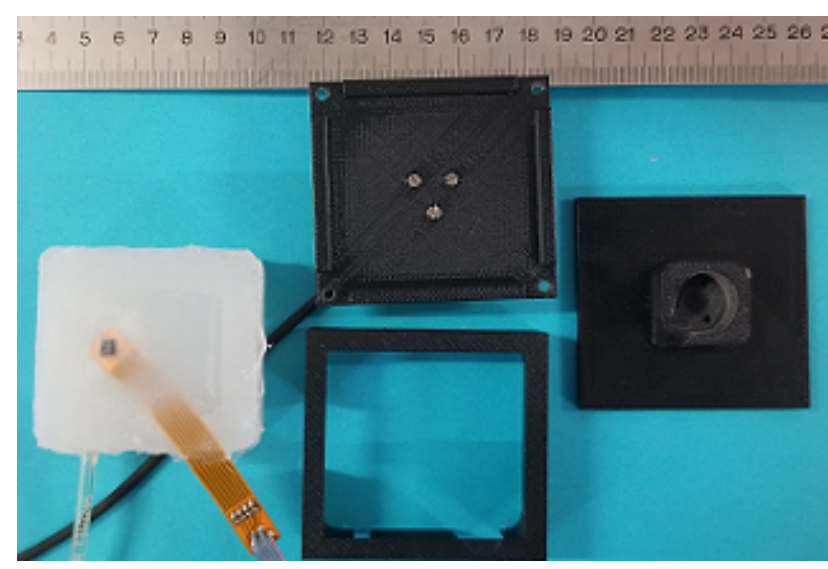

(a)

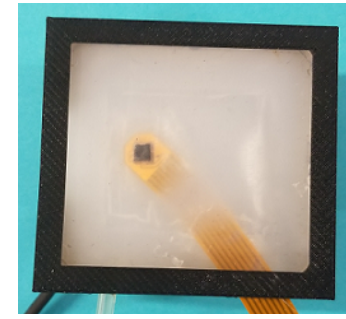

(b)

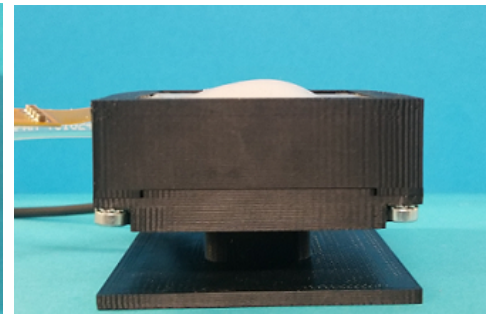

(c)
Fig. 5. Soft material tactile sensor with the fixing tool.

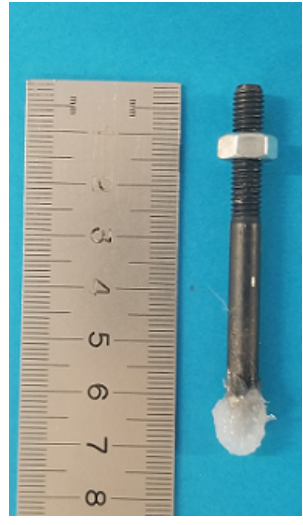

(a)

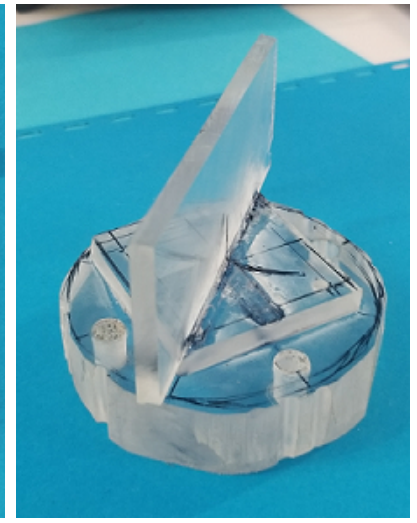

(b)
Fig. 6. End-effectors

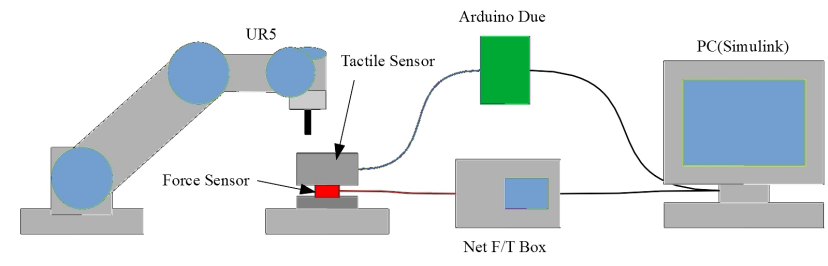

Fig. 7. Schematic drawing the experiment setup.

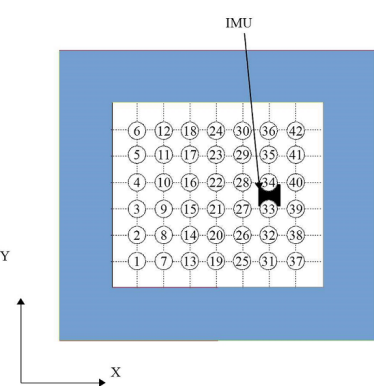

(a) 42 touching positions.

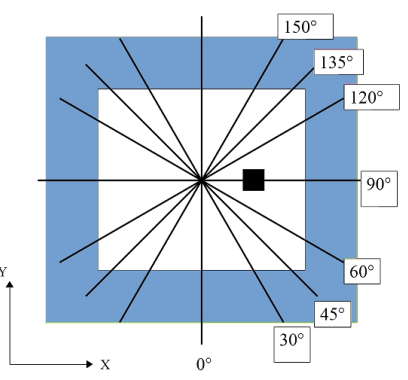

(b) Degree of rectangle.
Fig. 8. Experiment position.

by $10 \mathrm{ml}$ of air. In this way, the silicone skin changes its morphological structure and stiffness, according to the quantity of inflated air. Consequently, also the pose of the IMU will change accordingly with the silicone skin shape.

In this way, when a solid object get in contact with a point of the sensor's surface (i.e. the silicon skin), the pose of the IMU will change, based on the new distribution of the air, due to the deformation of the skin. The data related to the three-axis accelerometer the IMU is then acquired by means of an Arduino Due board, which is connected to a computer that collects all the data. In order to obtain the detection of the contact points on the sensor, the IMU's accelerometer data is processed by means of ANN.

ANN Structure: The ANN used in this study is a shallow feedforward network [12] implemented in Matlab. In particular, the network is used in order to perform data pattern classification, with the aim of detecting contact locations on the sensor surface, as reported in detail in the following section. With regard to the structure of the implemented ANN, an hyperbolic tangent sigmoid has been used for the activation functions of the hidden layers, whereas a normalized exponential for the output layer's activation function [13] - the latter used in order to map the non-normalized outputs of the previous layer to the probabilities of the different classes to be predicted. The number of hidden layers has been varied between different values in order to evaluate the performance of the classification - refer to Subsec. III-C. Therefore, first of all, the ANN is systematically trained on a series of contact points, as explained in the subsequent Sec. III.

\section{EXPERIMENTS AND RESULTS}

\section{A. Experimental Setup}

In order to train the $\mathrm{ANN}$ and perform the evaluation experiments, we equipped the tactile sensor described in the previous section by means of a rigid fixing support and a F/T sensor of the related vertical force of the contact.

The fixing support is realized by $3 \mathrm{D}$ printing (printer: Dimension Elite), and its structure can be observed in Fig. 5. In particular, Fig. 5(a) shows the different components of the fixing support, together with the soft tactile sensor, whereas Fig. 5(b) and 5(c) shows the final assembling in the upper and later views. Importantly, the fixing support embeds on its 
bottom the F/T sensor (Nano17, ATI Industrial Automation, Inc. ${ }^{4}$ ). The F/T sensor is used as measurement ground-truth the experiment in order to identify different contact conditions based on the measurement of the applied force through the proposed sensor.

A suitable set of contact forces are applied on a grid of contact points by means of two different tools shown in Fig. 6. Specifically, the tool reported in Fig. 6(a) is used to provide a single-point contact on the sensor surface, and it is covered on the tip by a thin silicone rubber layer; this silicon cover is necessary in order to reduce the sharpness of the metal stick and not damage the silicone skin of the sensor. On the other hand, the tool illustrated in Fig. 6(b) is used in order to apply to the tactile sensor a linear contact region, with a thickness of $4 \mathrm{~mm}$. These two tools are therefore mounted as end-effectors of a robotic manipulator (UR5, Universal Robots), and the training of the ANN and the related experiments are carried as explained in the following subsection.

\section{B. Experimental Protocol}

As illustrated in Fig. 7, the overall setup used during the experiment is composed by the UR5 manipulator controlled by ROS, the F/T sensor attached to base of the tactile sensor and the proposed tactile sensor. The F/T measurements are collected in Matlab/Simulink through a specific "Net F/T Box" connect to PC via Ethernet, while the IMU data coming from the developed sensor are collected by the same PC and Matlab/Simulink application by means of an Arduino Due USB interface.

This setup is used in order to carry out the following experimental protocol. Three different types of data acquisition are performed. The first data acquisition is composed by the output of the IMU while the UR5 is pressing on its surface with the metal stick end-effector (Fig. 6(a)) on a grid on 42 different locations, as illustrated in Fig. 8(a) (namely: the 42-locations-session); the data acquired during this process from the tactile sensor are labeled based on the Cartesian position of the robot, therefore associating the signals with 42 different classes. The second data acquisition is related to the IMU data when the robot is pressing on the tactile sensor by means of the linear-like end-effector of Fig. 6(b), applying the orientations of $0^{\circ}, 30^{\circ}, 60^{\circ}, 90^{\circ}, 120^{\circ}$ and $150^{\circ}$ (see Fig. 8(b)) (namely: the 6-orientations-session); in this case, the signals are labeled according to 6 classes, that corresponds to the six orientations of the linear region of contact points. Finally, the third data acquisition is built in the same way of the second, but considering the orientations of the linear region of contact points related to $0^{\circ}, 45^{\circ}, 90^{\circ}$ and $135^{\circ}$ (see Fig. 8(b)) (namely: the 4-orientations-session), corresponding to the labeling of the signals according to 4 classes. For each type of data acquisition, we repeated the experiment two times, and, for each of this repetition, we acquired the data for 3 levels of vertical force applied on the tactile sensor $-0.5 \mathrm{~N}$, $1 \mathrm{~N}$ and $2 \mathrm{~N}$ (using the information from the force sensor at

\footnotetext{
${ }^{4}$ www.ati-ia.com
}

the base of the tactile sensor) - and 3 levels of inflating air $-5 \mathrm{ml}, 7 \mathrm{ml}$ and $10 \mathrm{ml}$ (measured by using a syringe.) In this way, we obtained a total amount of 54 datasets (27 datasets for the first session, and 27 datasets for the second session.)

\section{Results}

After the experiment, we processed the data in order to properly obtain the aforementioned datasets and related labels. Then we trained and tested the ANN on the datasets of the first experimental session. In this case, the procedure for the training and testing of the ANN is realized for each combination of inflated air and vertical force levels, by means of a nested cross-validation (CV). In detail, the $\mathrm{CV}$ is composed by two nested loops. The inner loop consisted of a 10 -fold $\mathrm{CV}$, whereas the outer loop, a 10-fold CV as well, evaluated the performance of the ANN model that won in the inner loop, tested on a separated external fold. This validation process is then repeated for 9 different values of the number of hidden layers of the $\operatorname{ANN}\left(2^{0}, 2^{1}, 2^{2}, \ldots, 2^{8}\right)$, and the related accuracy results fo the ANN are reported in Fig. 9(a) for the 42-locations-session, in Fig. 9(d) for the 6orientations-session and in Fig. 9(g) for the 4-orientationssession. According to these figures, it is possible to see that the best accuracy for the 42-locations-session $(97.97 \%)$ is obtained for the combination of 1 hidden layer, $5 \mathrm{ml}$ of air and $2 \mathrm{~N}$ of vertical force; with regard to the 6-orientations-session, the best accuracy is obtained with the same combination (85.7\%); differently, for the 4-orientations-session the best accuracy $(85.7 \%)$ corresponded to 256 hidden layers, $10 \mathrm{ml}$ of air and $2 \mathrm{~N}$ of vertical force.

Additionally, for each type of data acquisition, the ANN trained on the data of the first session is tested on the data obtained from the second session, in order to evaluate the accuracy on datasets totally independent from the training set, as can be observed in the results reported in Fig. 9(b) for the 42-locations-session, in Fig. 9(e) for the 6-orientationssession and in Fig. 9(h) for the 4-orientations-session. Finally, Fig. 9(c), Fig. 9(f) and Fig. 9(i) reports the mean accuracy between the results obtained from the first and second session testing, obtaining: (i) for the 42-locations-session, the best mean accuracy $(84.12 \%)$ for the combination of 1 hidden layer, $5 \mathrm{ml}$ of air and $1 \mathrm{~N}$ of vertical force; (ii) for the 6-orientationssession, a best mean accuracy of $80.58 \%$ for 256 hidden layers, $10 \mathrm{ml}$ of air and $2 \mathrm{~N}$ of vertical force; and finally, (iii) for the 4-orientations-session, the combination of 256 hidden layers, $10 \mathrm{ml}$ of air and $2 \mathrm{~N}$ of vertical force, a mean accuracy of $96.97 \%$ is obtained.

\section{CONSLusions}

In this paper, the implementation and the experimental validation of a tactile sensor based on an inflatable chamber and an IMU is reported. The working principle of the sensor is based on the measurement through the IMU of the changes of the sensor surface due to the contacts with external objects. The proposed sensor is conceived to deal with both very soft deformable object as well as stiff objects thanks to 


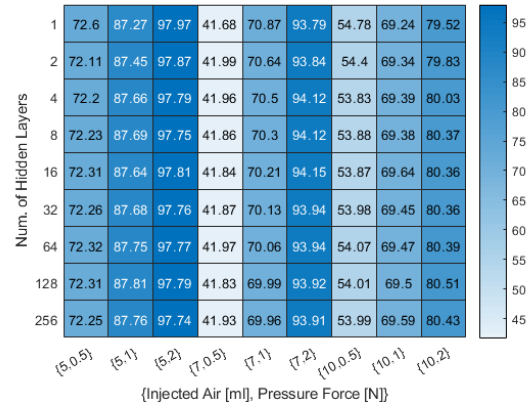

(a)

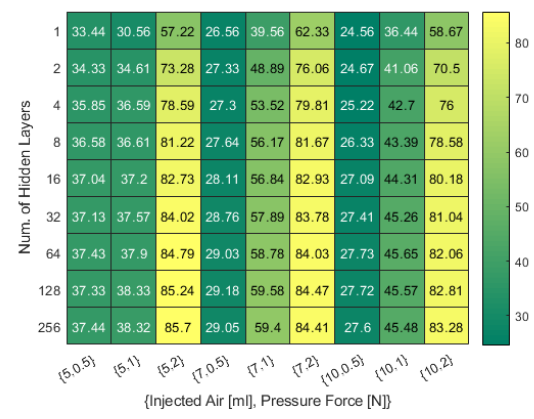

(d)

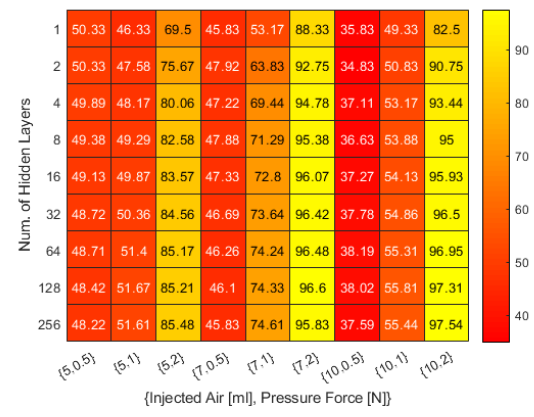

(g)

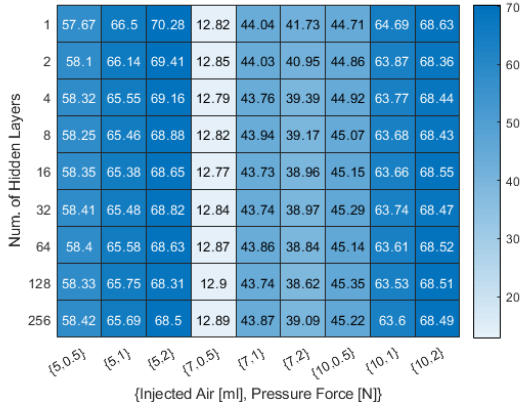

(b)

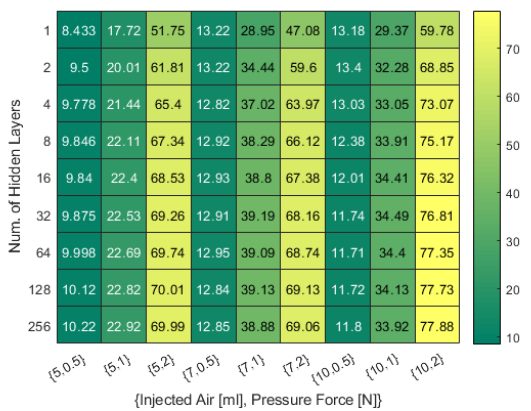

(e)

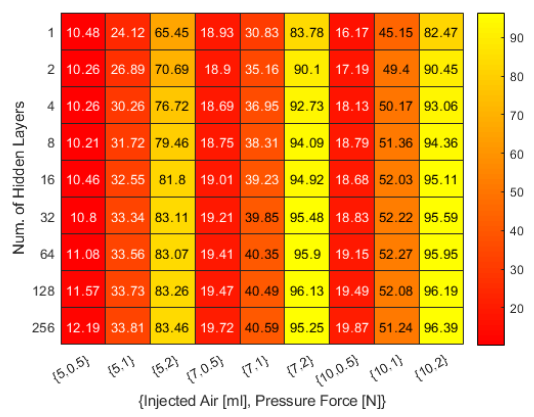

(h)

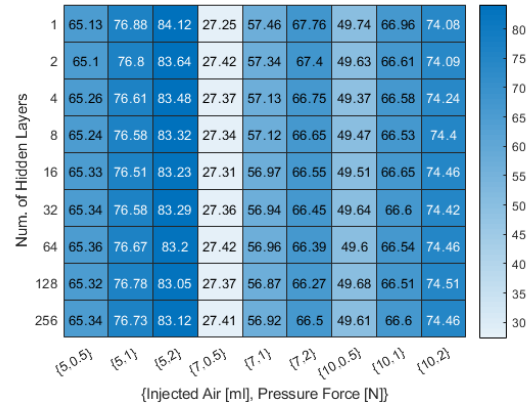

(c)

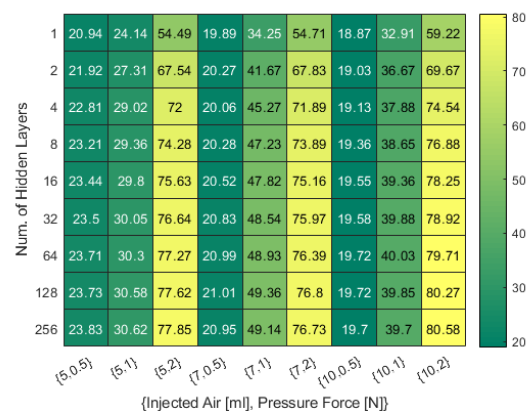

(f)

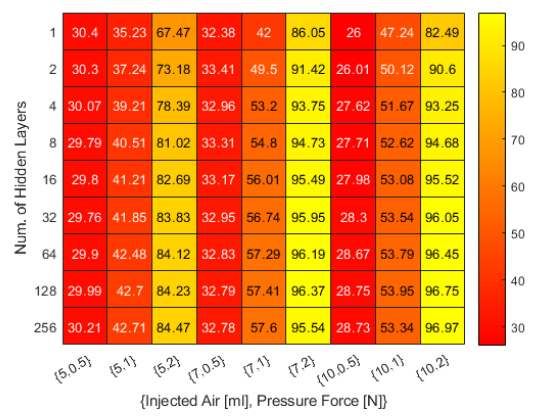

(i)

Fig. 9. Accuracies resulting from the ANN training and test for the different combinations of number of hidden layers and injected air-pressure force. Fig. 9(a) reports the results of the nested-CV process (first session dataset) for the 42-locations-session, Fig. 9(d) for the 6-orientations-session and Fig. 9(a) for the 4-orientations-session. Fig. 9(b) reports the results of the testing of the ANN on the trainingset-independent-dataset (second session dataset) for the 42-locations-session, Fig. 9(e) for the 6-orientations-session and Fig. 9(h) for the 4-orientations-session. Finally, Fig. 9(c), Fig. 9(f) and Fig. 9(i) reports the mean accuracy with respect to the first and second session datasets, for the 42-locations-session, 6-orientations-session and 4-orientations-session, respectively.

the possibility of changing the sensor responsiveness and sensitivity by changing the pressure inside the air chamber.

The calibration of the sensor both in terms of contact force detection, location of point contacts and orientation of line contacts has been executed by means of an artificial neural network, showing an accuracy ranging from $80.58 \%$ in the worst case to $96.97 \%$ in the best one.

Future works will be devoted to the application of the proposed sensor into a manipulation systems for soft objects. In this case the use of an additional IMU to compensate the motion of the sensor and of the whole manipulation system will be investigated in order to preserved the accuracy observed during the experiments reported in this paper.

\section{REFERENCES}

[1] F. Iida and C. Laschi, "Soft robotics: challenges and perspectives," Procedia Computer Science, vol. 7, pp. 99-102, 2011.

[2] S. Kim, C. Laschi, and B. Trimmer, "Soft robotics: a bioinspired evolution in robotics," Trends in biotechnology, vol. 31, no. 5, pp. 287294, 2013.

[3] D. Trivedi, C. D. Rahn, W. M. Kier, and I. D. Walker, "Soft robotics: Biological inspiration, state of the art, and future research," Applied bionics and biomechanics, vol. 5, no. 3, pp. 99-117, 2008.

[4] Z. Wang, Y. Torigoe, and S. Hirai, "A prestressed soft gripper: design, modeling, fabrication, and tests for food handling," IEEE Robotics and Automation Letters, vol. 2, no. 4, pp. 1909-1916, 2017.

[5] R. Pfeifer, M. Lungarella, and F. Iida, "Self-organization, embodiment, and biologically inspired robotics," science, vol. 318 , no. 5853, pp. 1088-1093, 2007.

[6] A. Kadowaki, T. Yoshikai, M. Hayashi, and M. Inaba, "Development of soft sensor exterior embedded with multi-axis deformable tactile sensor system," in RO-MAN 2009-The 18th IEEE International Symposium on 
Robot and Human Interactive Communication. IEEE, 2009, pp. 10931098.

[7] T. Paulino, P. Ribeiro, M. Neto, S. Cardoso, A. Schmitz, J. Santos-Victor, A. Bernardino, and L. Jamone, "Low-cost 3-axis soft tactile sensors for the human-friendly robot vizzy," in 2017 IEEE International Conference on Robotics and Automation (ICRA). IEEE, 2017, pp. 966-971.

[8] F. L. Hammond, Y. Mengüç, and R. J. Wood, "Toward a modular soft sensor-embedded glove for human hand motion and tactile pressure measurement," in 2014 IEEE/RSJ International Conference on Intelligent Robots and Systems. IEEE, 2014, pp. 4000-4007.

[9] H. Yamashita, Z. Wang, S. Hirai, K. Shibuya et al., "Wrin'tac: Tactile sensing system with wrinkle's morphological change," IEEE Transactions on Industrial Informatics, vol. 13, no. 5, pp. 2496-2506, 2017.

[10] H. X. Trinh, V. A. Ho, and K. Shibuya, "Computational model for tactile sensing system with wrinkle's morphological change," Advanced Robotics, vol. 32, no. 21, pp. 1135-1150, 2018.

[11] K. Shibuya, Y. Iwamoto, H. X. Trinh et al., "Detecting sliding movement location on morphologically changeable soft tactile sensing system with three-axis accelerometer," in 2019 2nd IEEE International Conference on Soft Robotics (RoboSoft). IEEE, 2019, pp. 337-342.

[12] H. Abdi, "A neural network primer," Journal of Biological Systems, vol. 2, no. 03, pp. 247-281, 1994

[13] M. Caudill and C. Butler, "Understanding neural networks: Computer explorations, vols. 1 and 2," 1992. 